\title{
YAP1-fusions in pediatric NF2-wildtype meningioma
}

Sievers, Philipp ; Chiang, Jason ; Schrimpf, Daniel ; Stichel, Damian ; Paramasivam, Nagarajan ; Sill, Martin ; et al ; Rushing, Elisabeth

DOI: https://doi.org/10.1007/s00401-019-02095-9

Posted at the Zurich Open Repository and Archive, University of Zurich ZORA URL: https://doi.org/10.5167/uzh-178933

Journal Article

Accepted Version

Originally published at:

Sievers, Philipp; Chiang, Jason; Schrimpf, Daniel; Stichel, Damian; Paramasivam, Nagarajan; Sill, Martin; et al; Rushing, Elisabeth (2019). YAP1-fusions in pediatric NF2-wildtype meningioma. Acta Neuropathologica, 139(1):215-218.

DOI: https://doi.org/10.1007/s00401-019-02095-9 
2 Philipp Sievers ${ }^{1,2}$, Jason Chiang $^{3}$, Daniel Schrimpf ${ }^{1,2}$, Damian Stichel ${ }^{1,2}$, Nagarajan

3 Paramasivam ${ }^{4}$, Martin Sill ${ }^{5,6}$, Tenzin Gayden ${ }^{7,8}$, Belen Casalini $^{1,2}$, David E. Reuss ${ }^{1,2}$, James

4 Dalton $^{3}$, Kristian W. Pajtler ${ }^{5,6,9}$, Daniel Hänggi ${ }^{10}$, Christel Herold-Mende ${ }^{11}$, Elisabeth

5 Rushing ${ }^{12}$, Andrey Korshunov ${ }^{1,2,5}$, Christian Mawrin ${ }^{13}$, Michael Weller ${ }^{14}$, Matthias Schlesner ${ }^{4}$,

6 Wolfgang Wick ${ }^{15,16}$, Nada Jabado ${ }^{7,8}$, David T.W. Jones ${ }^{5,17}$, Stefan M. Pfister ${ }^{5,6,9}$, Andreas von

7 Deimling ${ }^{1,2}$, David W. Ellison ${ }^{3}$, Felix Sahm ${ }^{1,2,5}$

$9{ }^{1}$ Department of Neuropathology, Institute of Pathology, University Hospital Heidelberg, Heidelberg, Germany

$10{ }^{2}$ Clinical Cooperation Unit Neuropathology, German Consortium for Translational Cancer Research (DKTK), German Cancer

11 Research Center (DKFZ), Heidelberg, Germany

$12{ }^{3}$ Department of Pathology, St. Jude Children's Research Hospital, Memphis, TN, USA

$13{ }^{4}$ Bioinformatics and Omics Data Analytics, German Cancer Research Center (DKFZ), Heidelberg, Germany

$14{ }^{5}$ Hopp Children's Cancer Center Heidelberg (KiTZ), Heidelberg, Germany

15 'Division of Pediatric Neurooncology, German Cancer Consortium (DKTK), German Cancer Research Center (DKFZ),

16 Heidelberg, Germany

17 'Department of Human Genetics, McGill University, Montreal, QC, H3A 1B1, Canada

18 'Department of Pediatrics, McGill University, and The Research Institute of the McGill University Health Center, Montreal, QC, 19 H4A 3J1, Canada

20 'Department of Pediatric Oncology, Hematology, Immunology and Pulmonology, University Hospital Heidelberg, Heidelberg, 21 Germany

$22{ }^{10}$ Department of Neurosurgery, University Medical Centre Mannheim, University of Heidelberg, Mannheim, Germany

$23{ }^{11}$ Division of Experimental Neurosurgery, Department of Neurosurgery, University Hospital Heidelberg, Heidelberg, Germany

$24 \quad{ }^{12}$ Institute of Neuropathology, University Hospital and University of Zurich, Zurich, Switzerland

$25{ }^{13}$ Institute of Neuropathology, Otto-von-Guericke University, Magdeburg, Germany

$26{ }^{14}$ Department of Neurology, University Hospital and University of Zurich, Zurich, Switzerland

$27{ }^{15}$ Clinical Cooperation Unit Neurooncology, German Consortium for Translational Cancer Research (DKTK), German Cancer

28 Research Center (DKFZ), Heidelberg, Germany

${ }^{16}$ Department of Neurology and Neurooncology Program, National Center for Tumor Diseases, Heidelberg University Hospital, Heidelberg, Germany

${ }^{17}$ Pediatric Glioma Research Group, German Cancer Research Center (DKFZ), Heidelberg, Germany

Corresponding author:

Felix Sahm, MD, PhD

Department of Neuropathology

University Hospital Heidelberg

69120 Heidelberg, Germany

Fon: +49-6221 56-37886

Fax: +49-622156 4566

felix.sahm@med.uni-heidelberg.de

Key words: pediatric meningioma, molecular diagnostics, gene fusions, RNA sequencing, 
Meningioma is the most common primary central nervous system (CNS) tumor [8]. In contrast to adulthood, meningiomas are rare among children and adolescents and frequently (about 38\%) occur in the context of tumor predisposition syndromes [12]. In line with the 4 frequent inactivation of NF2 in adult meningiomas, neurofibromatosis type 2 is the most common inherited syndrome predisposing to the early development of meningiomas, which are often multiple. Other germline alterations predisposing to meningioma development are SMARCE1 [14] and SUFU mutations [1]. More recently identified drivers of meningiomas include AKT1/TRAF7, SMO, KLF4/TRAF7, and PIK3CA mutations [3, 5].

The mutational underpinnings of sporadic pediatric meningioma have remained elusive to date. We report in-frame gene rearrangements predicted to result in fusions involving YAP1 in nine meningiomas. We initially identified a YAP1-MAML2 fusion by clinical RNA sequencing in a four year old female patient with an intraventricular mass, histologically compatible with meningioma. Subsequently, based on our database of $>30,000$ DNA methylation profiles of brain tumors [4], including $>1,000$ meningiomas (among them about 102 pediatric meningiomas, defined as age of diagnosis equal or below 18 years) [13], and corresponding copy number information, we additionally identified eight meningiomas with structural alterations affecting chromosome 11q around the YAP1 locus (Online Resources Supplementary Fig. 2 and 3). All clustered with reference meningioma cases in t-SNE analysis of DNA methylation data (Fig. 1a) and showed histological and immunohistochemical features of meningioma (Online Resource Supplementary Fig. 1), despite two being initially diagnosed as glioma. Interestingly, of the nine cases two were classified as transitional and two as atypical meningiomas - both subtypes which often carry NF2 mutations. With the presented case number and incomplete information on few samples, robust conclusions are, however, not derivable on subtype distribution. This limitation also applies to localizations. The clinicopathological characteristics are summarized in Table 1. RNA (seven samples) or exome (one sample) sequencing [15] revealed the presence of YAP1 fusions in all eight additional tumors (Fig. 1b-d and Online Resource Supplementary Table 1).

Seven tumors harbored a rearrangement of YAP1-MAML2 involving exons 1-5 $(n=5)$ or only in exon $1(\mathrm{n}=2)$ of YAP1 (NM_001130145) and exon 2-5 of MAML2 (NM_032427). YAP1MAML2 fusions were verified by fluorescence in situ hybridization (FISH) performed in two cases (Online Resource Supplementary Fig. 4). A YAP1-PYGO1 fusion was seen in a single case, containing exons 1-4 of YAP1 and exons 2-3 of PYGO1 (NM_015617). Additionally, a YAP1-LMO1 fusion was detected in another case involving exons 1-4 of YAP1 and exons 24 of LMO1. Of note, seven of the additional eight patients were children or adolescents, whereas one patient was an adult.

YAP1 is a transcriptional co-activator and downstream effector of the HIPPO pathway that acts mainly through TEAD family transcription factors and regulates expression of genes involved in cell proliferation and apoptosis [6, 7, 19, 20]. Deregulation of the HIPPO pathway via overexpression of $Y A P 1$, leading to tumorigenesis is a frequent event in human malignancies including meningiomas [2, 9]. Rearrangements involving the YAP1 gene have also recently been implicated as a driver in different types of cancer. Valouev et al. reported an in-frame gene fusion between $Y A P 1$ and MAML2 in nasopharyngeal carcinomas [16]. A similar fusion between YAP1 and MAMLD1 has been described in ependymoma [10, 11]. Both MAML2 and MAMLD1 are members of the Mastermind gene family and act as transcriptional co-activators of $\mathrm{NOTCH}$ signaling [17]. The YAP1-MAML2 rearrangement combines the transcriptional activation domain of MAML2 with the TEAD-binding domain of 
YAP1, which likely results in NOTCH-independent co-activation of TEAD-mediated HIPPO signaling [17]. While PYGO1 has been associated with different types of cancer, structural rearrangements including YAP1 and PYGO1 have not been reported to the best of our knowledge. Notably, PYGO1 also functions as a transcriptional co-activator in the Wnt pathway. LMO1 acts as a transcriptional regulator with a tumor-promoting activity, but its role in tumors has not been well studied.

These alterations seem to act as an alternative to NF2 inactivation, since no NF2 alterations were detected in the present cohort. The NF2 gene product, the tumor suppressor merlin, functions upstream of the HIPPO pathway and there is growing evidence suggesting a functional link between NF2, YAP1 and activation of the HIPPO pathway [2, 18]. In line, YAP1 fusion positive meningiomas clustered closer to NF2 mutant cases than other pediatric meningiomas (Online Resource Supplementary Fig. 5). This observation parallels the virtually mutually exclusive alterations of NF2 and YAP1 in ependymoma. Since DNA methylation correlates with cell-of-origin, the proximity of YAP1- and NF2-altered cases in the clustering might indicate more similarities of their precursor cells compared to SMARCE1 mutant meningiomas. However, the lack of AKT1, SMO, or KLF4/TRAF7 mutant pediatric meningiomas and the few SMARCE1 cases are limitations of this analysis.

Our findings identify YAP1 fusions as a potential oncogenic driver in the development of meningiomas, predominantly in pediatric patients, and strengthen the hypothesis that deregulation of the HIPPO pathway is a central mechanism in meningioma tumorigenesis. Further studies in larger cohorts are needed to determine additional downstream functional consequences and a possible prognostic role of YAP1 alterations in meningiomas.

\section{Acknowledgements}

We thank L. Dörner and H. Y. Nguyen for skillful technical assistance and the microarray unit of the DKFZ Genomics and Proteomics Core Facility for providing Illumina DNA methylation array-related services. This study was supported by the German Cancer Aid (70112956) and Else Kröner-Fresenius Stiftung (EKFS 2015_A60). FS is a fellow of the Else Kröner Excellence Program of the Else Kröner-Fresenius Stiftung (EKFS 2017_EKES.24).

\section{Figure legends}

Fig. 1 a) Unsupervised hierarchical clustering of DNA methylation profiles in nine YAP1-fused meningiomas (MNG_YAP1) alongside 128 well-characterized CNS neoplasms and control tissue shown in a two-dimensional representation of pairwise sample correlations using the 15,000 most variant probes by t-distributed stochastic neighbor embedding (t-SNE) dimensionality reduction. Reference methylation classes: ependymoma, posterior fossa group A (EPN_PFA), ependymoma, posterior fossa group B (EPN_PFB), ependymoma, RELA fusion (EPN_RELA), ependymoma, YAP fusion (EPN_YAP), subependymoma, posterior fossa (EPN_PF_SE), ependymoma, spinal (EPN_SPINE), subependymoma, spinal (EPN_SPINE_SE), subependymoma, supratentorial (EPN_ST_SE), meningioma (MNG), chordoma (CHORDM), melanoma (MELN), schwannoma (SCHW) and control tissue white matter (CONTROL). Schematic of the YAP1-MAML2 fusion involving exons 1-5 of YAP1 and exons 2-5 of MAML (b), the YAP1-PYGO1 fusion involving exons 1-4 of YAP1 and exons 2-3 of MAML2 (c) and the YAP1-LMO1 fusion involving exons 1-4 of YAP1 and exons 2-4 of LMO1 (d). 
Table1 Clinicopathological characteristics of the YAP1-fused meningioma cohort

\begin{tabular}{cccccc}
\hline Case \# & $\begin{array}{c}\text { Age } \\
\text { (years) }\end{array}$ & Sex & Tumor location & $\begin{array}{c}\text { Initial } \\
\text { Diagnosis }\end{array}$ & Genetic alteration \\
\hline 1 & 4 & $\mathrm{~F}$ & lateral ventricles, 3rd ventricle & MNG & YAP1:MAML2 \\
2 & 3 & $\mathrm{M}$ & temporal & $\mathrm{PXA}$ & YAP1:PYGO1 \\
3 & 1 & $\mathrm{M}$ & 3rd ventricle, lateral ventricle & $\mathrm{pHGG}$ & YAP1:MAML2 \\
4 & 2 & $\mathrm{M}$ & skull base & MNG & YAP1:MAML2 \\
5 & 36 & $\mathrm{M}$ & optic nerve & MNG & YAP1:MAML2 \\
6 & 8 & $\mathrm{~F}$ & skull base (supra-/infratentorial) & MNG & YAP1:LMO1 \\
7 & 17 & $\mathrm{M}$ & cavernous sinus & MNG & YAP1:MAML2 \\
8 & 7 & $\mathrm{~F}$ & parietal & MNG & YAP1:MAML2 \\
9 & 7 & $\mathrm{~F}$ & frontal & MNG & YAP1:MAML2 \\
\hline
\end{tabular}

MNG - meningioma, PXA - pleomorphic xanthoastrocytoma, pHGG - pediatric high grade glioma, $\mathrm{F}$ - female, $\mathrm{M}$ subtypes meningiomas and WHO grade are provided in Supplementary Table 2. 


\section{References}

1 Aavikko M, Li SP, Saarinen S, Alhopuro P, Kaasinen E, Morgunova E, Li Y, Vesanen K, Smith MJ, Evans DGet al (2012) Loss of SUFU function in familial multiple meningioma. Am J Hum Genet 91: 520-526 Doi 10.1016/j.ajhg.2012.07.015

2 Baia GS, Caballero OL, Orr BA, Lal A, Ho JS, Cowdrey C, Tihan T, Mawrin C, Riggins GJ (2012) Yes-associated protein 1 is activated and functions as an oncogene in meningiomas. Mol Cancer Res 10: 904-913 Doi 10.1158/1541-7786.MCR-12-0116

3 Brastianos PK, Horowitz PM, Santagata S, Jones RT, McKenna A, Getz G, Ligon KL, Palescandolo E, Van Hummelen P, Ducar MDet al (2013) Genomic sequencing of meningiomas identifies oncogenic SMO and AKT1 mutations. Nat Genet 45: 285-289 Doi $10.1038 / n g .2526$

4 Capper D, Jones DTW, Sill M, Hovestadt V, Schrimpf D, Sturm D, Koelsche C, Sahm F, Chavez L, Reuss DEet al (2018) DNA methylation-based classification of central nervous system tumours. Nature 555: 469-474 Doi 10.1038/nature26000

Clark VE, Erson-Omay EZ, Serin A, Yin J, Cotney J, Ozduman K, Avsar T, Li J, Murray PB, Henegariu Oet al (2013) Genomic analysis of non-NF2 meningiomas reveals mutations in TRAF7, KLF4, AKT1, and SMO. Science 339: 1077-1080 Doi 10.1126/science.1233009 Levy D, Adamovich Y, Reuven N, Shaul Y (2008) Yap1 phosphorylation by c-Abl is a critical step in selective activation of proapoptotic genes in response to DNA damage. Mol Cell 29: 350-361 Doi 10.1016/j.molcel.2007.12.022

Liu-Chittenden Y, Huang B, Shim JS, Chen Q, Lee SJ, Anders RA, Liu JO, Pan D (2012) Genetic and pharmacological disruption of the TEAD-YAP complex suppresses the oncogenic activity of YAP. Genes Dev 26: 1300-1305 Doi 10.1101/gad.192856.112 Louis DN, Ohgaki H, Wiestler OD, Cavenee WK (2016) WHO Classification of Tumours of the Central Nervous System. IARC, City A, Muir B, Li W, Sgroi DC, Deng CX, Brugge JS, Haber DA (2006) Transforming properties of YAP, a candidate oncogene on the chromosome 11q22 amplicon. Proc Natl Acad Sci U S A 103: 12405-12410 Doi 10.1073/pnas.0605579103

Pajtler KW, Witt H, Sill M, Jones DT, Hovestadt V, Kratochwil F, Wani K, Tatevossian R, Punchihewa C, Johann Pet al (2015) Molecular Classification of Ependymal Tumors across All CNS Compartments, Histopathological Grades, and Age Groups. Cancer Cell 27: 728-743 Doi 10.1016/j.ccell.2015.04.002

Parker M, Mohankumar KM, Punchihewa C, Weinlich R, Dalton JD, Li Y, Lee R, Tatevossian RG, Phoenix TN, Thiruvenkatam Ret al (2014) C11orf95-RELA fusions drive oncogenic NFkappaB signalling in ependymoma. Nature 506: 451-455 Doi 10.1038/nature13109

Pathmanaban ON, Sadler KV, Kamaly-AsI ID, King AT, Rutherford SA, Hammerbeck-Ward C, McCabe MG, Kilday JP, Beetz C, Poplawski NKet al (2017) Association of Genetic Predisposition With Solitary Schwannoma or Meningioma in Children and Young Adults. JAMA Neurol 74: 1123-1129 Doi 10.1001/jamaneurol.2017.1406

Sahm F, Schrimpf D, Stichel D, Jones DTW, Hielscher T, Schefzyk S, Okonechnikov K, Koelsche C, Reuss DE, Capper Det al (2017) DNA methylation-based classification and grading system for meningioma: a multicentre, retrospective analysis. Lancet Oncol 18: 682694 Doi 10.1016/S1470-2045(17)30155-9

14 Smith MJ, O'Sullivan J, Bhaskar SS, Hadfield KD, Poke G, Caird J, Sharif S, Eccles D, Fitzpatrick D, Rawluk Det al (2013) Loss-of-function mutations in SMARCE1 cause an inherited disorder of multiple spinal meningiomas. Nat Genet 45: 295-298 Doi 10.1038/ng.2552

Stichel D, Schrimpf D, Casalini B, Meyer J, Wefers AK, Sievers P, Korshunov A, Koelsche C, Reuss DE, Reinhardt Aet al (2019) Routine RNA sequencing of formalin-fixed paraffinembedded specimens in neuropathology diagnostics identifies diagnostically and therapeutically relevant gene fusions. Acta neuropathologica: Doi 10.1007/s00401-01902039-3

Valouev A, Weng Z, Sweeney RT, Varma S, Le QT, Kong C, Sidow A, West RB (2014) Discovery of recurrent structural variants in nasopharyngeal carcinoma. Genome Res 24: 300309 Doi 10.1101/gr.156224.113

17 Wu L, Sun T, Kobayashi K, Gao P, Griffin JD (2002) Identification of a family of mastermindlike transcriptional coactivators for mammalian notch receptors. Mol Cell Biol 22: 7688-7700 Doi 10.1128/mcb.22.21.7688-7700.2002 
18 Zhang N, Bai H, David KK, Dong J, Zheng Y, Cai J, Giovannini M, Liu P, Anders RA, Pan D (2010) The Merlin/NF2 tumor suppressor functions through the YAP oncoprotein to regulate tissue homeostasis in mammals. Dev Cell 19: 27-38 Doi 10.1016/j.devcel.2010.06.015

19 Zhao B, Wei X, Li W, Udan RS, Yang Q, Kim J, Xie J, Ikenoue T, Yu J, Li Let al (2007) Inactivation of YAP oncoprotein by the Hippo pathway is involved in cell contact inhibition and tissue growth control. Genes Dev 21: 2747-2761 Doi 10.1101/gad.1602907

20 Zhao B, Ye X, Yu J, Li L, Li W, Li S, Yu J, Lin JD, Wang CY, Chinnaiyan AMet al (2008) TEAD mediates YAP-dependent gene induction and growth control. Genes Dev 22: 1962-1971 Doi 10.1101/gad.1664408 


\section{heiBOX}

Suppl. material YAP1_new.pdf

Aktueller Pfad: YAP1 / Suppl. material YAP1_new.pdf

\section{Supplementary materials and methods}

\section{Sample collection}

Besides the index cases emerged in routine diagnostics, additional cases of YAP1-fused meningioma and reference cases were retrieved from the archives of the Departments of Neuropathology at the University Hospitals in Heidelberg, Magdeburg (both Germany) and Zürich (Switzerland), the Department of Pediatrics, McGill University, and The Research Institute of the McGill University Health Center, Montreal (Canada) as well as the Department of Pathology, St. Jude Children's Research Hospital, Memphis, TN (USA). Analysis of tissue and clinical data was performed in accordance with local ethical regulations.

\section{Immunohistochemistry}

Immunohistochemistry was performed on a Ventana BenchMark ULTRA Immunostainer (Ventana Medical Systems, Tucson, AZ, USA) for all cases with sufficient material $(n=5)$. Antibodies were directed against: epithelial membrane antigen (EMA; Clone GP1.4, mouse monoclonal, dilution 1:1000, Thermo Fisher Scientific, Fremont, CA, USA), somatostatin receptor 2A (SSTR2A; SS-8000-RM, rabbit monoclonal, dilution 1:10, Biotrend, Cologne, Germany) and Ki-67 (clone MIB-1, mouse monoclonal, 1:100 dilution, Dako Agilent, Santa Clara, CA, USA).

\section{Nucleic acid extraction, DNA methylation analysis and RNAlexome sequencing}

Representative tumor tissue with highest tumor cell content was histologically identified and selected for nucleic acid extraction using local standard protocols. DNA methylation profiling of all samples was performed using the Infinium MethylationEPIC (850k) BeadChip (Illumina, San Diego, CA, USA) or Infinium HumanMethylation450 (450k) BeadChip (Illumina) array according to the manufacturer's instructions. Filtering and genome-wide copy number analyses were performed as previously described, using the 'conumee' package in $R$ (http://www.bioconductor.org) [2]. RNA and DNA sequencing were performed on a NextSeq 500 (Illumina) as previously described [1]. Fusion discovery used defuse and arriba (https://github.com/suhrig/arriba/).

\section{Fluorescence in situ hybridization (FISH)}

FISH was performed using the YAP1 Break Apart Probe (Empire Genomics, Williamsville, NY, USA) on interphase nuclei on $5 \mu \mathrm{m}$ FFPE sections in two cases according to the standard procedures and manufacturer's instructions. The 5' centromeric and 3 ' telomeric probes are labeled with 5-carboxyl-x-rhodamine and 5-fluorescein, respectively. 
Seite 2 von 2

https://heibox.uni-heidelberg.de/d/8aeecb0583c14200bc51/files/?p=\%2FSuppl.\%20m... 20.11.2019 


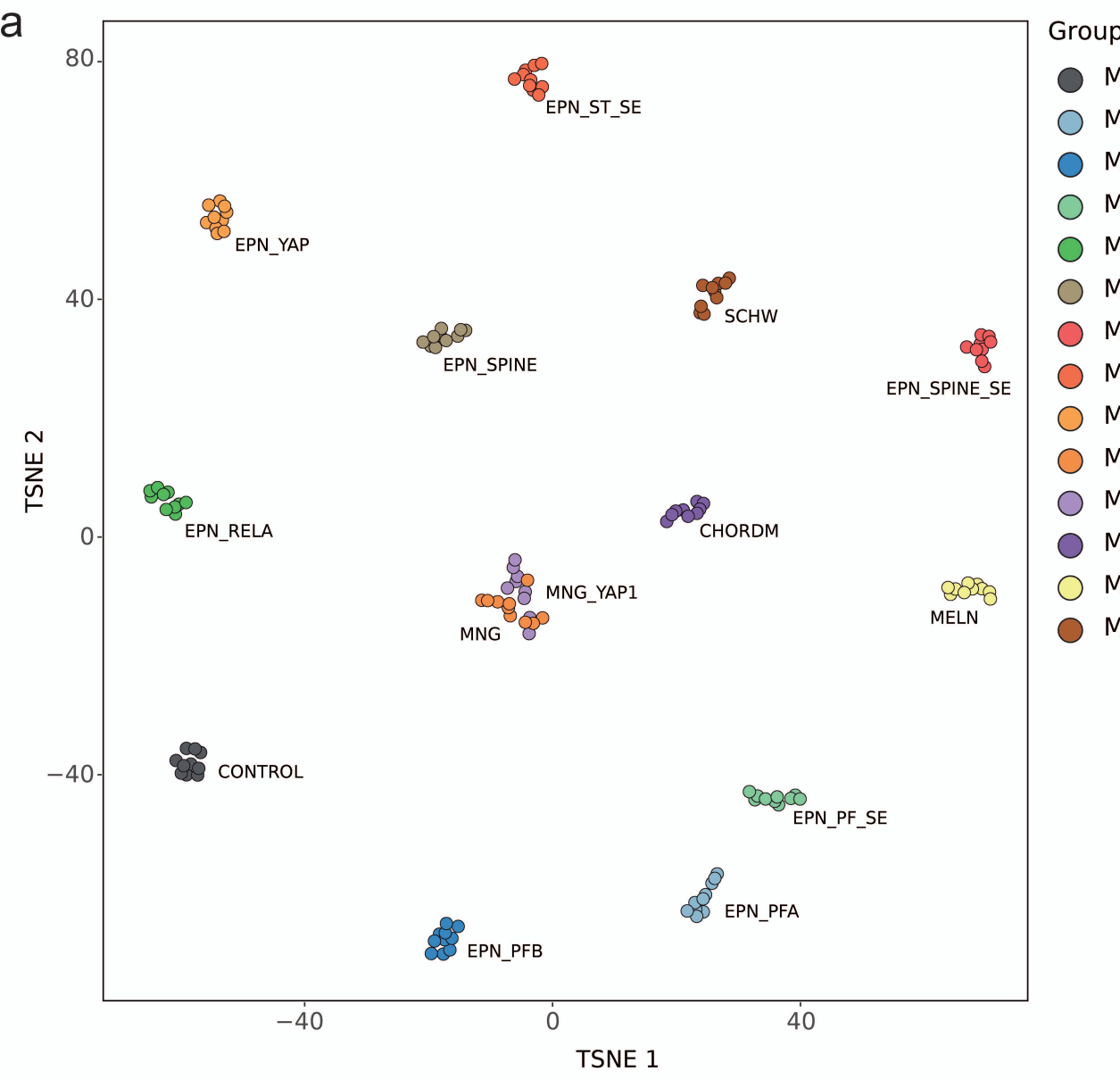

